\title{
A!
}

This is an electronic reprint of the original article.

This reprint may differ from the original in pagination and typographic detail.

Besenbacher, F.; Nørskov, J. K.; Puska, M. J.; Holloway, S.

\section{Excitation of Hydrogen Motion inside a Nickel Vacancy}

Published in:

Physical Review Letters

DOI:

10.1103/PhysRevLett.55.852

Published: 19/08/1985

Document Version

Publisher's PDF, also known as Version of record

Please cite the original version:

Besenbacher, F., Nørskov, J. K., Puska, M. J., \& Holloway, S. (1985). Excitation of Hydrogen Motion inside a Nickel Vacancy. Physical Review Letters, 55(8), 852-855. https://doi.org/10.1103/PhysRevLett.55.852

This material is protected by copyright and other intellectual property rights, and duplication or sale of all or part of any of the repository collections is not permitted, except that material may be duplicated by you for your research use or educational purposes in electronic or print form. You must obtain permission for any other use. Electronic or print copies may not be offered, whether for sale or otherwise to anyone who is not an authorised user. 


\title{
Excitation of Hydrogen Motion inside a Nickel Vacancy
}

\author{
F. Besenbacher \\ Institute of Physics, University of Aarhus, DK-8000 Aarhus C, Denmark \\ and \\ J. K. Nørskov \\ NORDITA, Blegdamsvej 17, DK-2100 Copenhagen $\varnothing$, Denmark \\ and \\ M. J. Puska \\ Laboratory of Physics Helsinki, University of Technology, SF-02150 Espoo, Finland \\ and \\ S. Holloway \\ The Donnan Laboratories, University of Liverpool, L69 3BX Liverpool, United Kingdom
}

(Received 26 April 1985)

\begin{abstract}
By means of the ion-channeling technique, we have shown the existence of a very low-energy $(\sim 3 \mathrm{meV})$ excitation for deuterium impurities trapped to vacancies in nickel. The excitation is associated with a change of the D location from near octahedral to near tetrahedral. On the basis of calculations of the $\mathrm{D}$ potentials within the effective-medium theory and the quantum treatment of the $\mathrm{D}$ motion, we suggest that the observed state can be regarded as an excited standing wave of a particle (D) on a sphere (internal surface around the vacancy).
\end{abstract}

PACS numbers: $61.80 . \mathrm{Mk}, 61.70 . \mathrm{Bv}, 61.70 . \mathrm{Tm}$

The interaction of hydrogen isotopes ${ }^{1}$ with defects in bulk metals has been the subject of an increasing number of detailed investigations (for reviews, see Besenbacher, Myers, and Nørskov ${ }^{2}$ and Myers et al. ${ }^{3}$ ), and very recently, new insight into the microscopic nature of the hydrogen-metal interaction has been gained from an interplay between experiment and theory. 4,5

In the present Letter, we consider D trapped to vacancies in nickel and demonstrate the existence of a very low-energy $(\sim 3 \mathrm{meV})$ excitation which is characterized by a tetrahedral-like D-lattice location, as opposed to the near-octahedral site of D in the ground state in the vacancy. We propose that this excitation corresponds to the motion of $\mathrm{D}$ around the vacancy. The results offer a new interpretation for other metalhydrogen systems such as, e.g., D trapped to vacancies in palladium.

The experimental setup and method have been previously detailed. ${ }^{6}$ The following is a brief description: $\mathrm{D}$ was implanted at $25 \mathrm{~K}$ into Ni single crystals along a random direction at an energy of $15 \mathrm{keV}$ and at a fluence of $6 \times 10^{14} \mathrm{D} / \mathrm{cm}^{2}$. The total amount and the depth distribution of $\mathrm{D}$ were monitored by ion-beam analysis detecting either protons or $\alpha$ particles from the $750-\mathrm{keV}{ }^{3} \mathrm{He}$-excited nuclear reaction $\mathrm{D}\left({ }^{3} \mathrm{He}, p\right) \alpha$. The D-lattice location was determined by the ionchanneling technique. ${ }^{7}$ The crystals were subjected to isochronal-annealing sequences of $15 \mathrm{~min}$ at tempera- tures ranging from 25 to $270 \mathrm{~K}$ and then cooled to the implant temperature of $25 \mathrm{~K}$, at which the analysis, i.e., the lattice location, was performed. The detailed analysis of the channeling results was performed by comparison of the measured angular yields with theoretical multirow continuum-model calculations.

An increase in annealing temperature will cause a shift in occupancy from solution to weaker and finally stronger traps, and after an anneal at $T_{a}=270 \mathrm{~K}, \mathrm{D}$ populates the strongest lattice-defect traps, i.e., vacancies exclusively. ${ }^{6}$ The corresponding $\mathrm{D}$ angular scan for the $\langle 100\rangle$ axis is shown by the open circles in Fig. 1. We have not been able to find a unique site that explains these channeling data. To account for the experimental results given by the open circles in Fig. 1, we assumed, for simplicity, two positions only, and the lower dashed curves in Fig. 1 is calculated for $87 \%$ of the D displaced $0.3 \AA$ from the octahedral $\left(O_{h}\right)$ site towards the vacancy, and the remaining $13 \%$ displaced $0.2 \AA$ from the tetrahedral $\left(T_{h}\right)$ site towards the vacancy.

In Fig. 2, we show results of a calculation of the binding energy of $\mathrm{D}$ in a $\mathrm{Ni}$ vacancy using the effective-medium theory. ${ }^{8}$ Also shown are the D ground-state densities, i.e., the square of the atom ground-state wave function which is obtained by solving the Schrödinger equation for a $\mathrm{D}$ atom moving in the calculated potential. ${ }^{9}$ The D atom is clearly delocalized but with clear maxima between the vacancy 


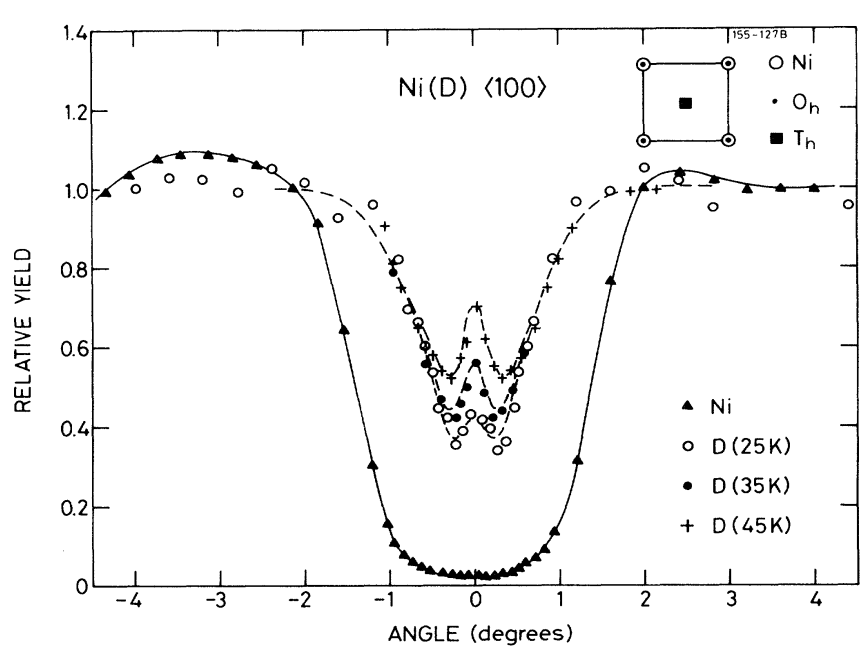

FIG. 1. Normalized channeling yields from $\mathrm{D}$ and $\mathrm{Ni}$ after annealing at $T_{a}=270 \mathrm{~K}$ for $15 \mathrm{~min}$. The measurements are carried out at 25,25 , and $45 \mathrm{~K}$, respectively, and are reversible with respect to temperature. In the top corner, the projection of the $\langle 100\rangle$ channel is shown, and it is seen that displaced $O_{h}$ and $T_{h}$ sites are uniquely separable from a $\langle 100\rangle$ axial scan since a $T_{h}$ site is located in the center of the $\langle 100\rangle$ axis, resulting in a flux peak, whereas the $O_{h}$ site is shadowed by the Ni rows of atoms, resulting in a channeling dip that is narrowed for a displaced $O_{h}$ site (Ref. 7). Channeling scans were carried out along other major axial and planar directions, and these results were consistent with the present $\langle 100\rangle$ results.

and the $O_{h}$ sites. Qualitatively, the maxima at displaced $O_{h}$ sites and the delocalized nature of the ground-state D density is consistent with the experimental results. Quantivatively, however, the D density in the region between the vacancy and the $T_{h}$ site is probably not sufficient to account for the $13 \% T_{h}$-like occupancy. The maxima of the displaced $O_{h}$ site is closer to the vacancy than the experimentally determined position by $\sim 0.65 \AA$, corresponding to a difference in D-Ni bond length of about $0.2 \AA$. This reflects inaccuracies in the calculated potential. ${ }^{6}$

After the annealing to $270 \mathrm{~K}$ and the measurement at $25 \mathrm{~K}$, we repeat this angular $\langle 100\rangle$ scan but now with the measuring temperature increased to 35 and 45 $\mathrm{K}$, respectively. The results are shown again in Fig. 1 . it is seen that the $T_{h}$ occupancy represented by the flux peak increases with temperature. This indicates that the $T_{h}$-like component must be an excited state which is increasingly populated with increasing temperature. These higher-temperature results were analyzed, once again with the assumption of only a displaced $O_{h}$ and a displaced $T_{h}$ site; the dashed curves through the experimental data are the results of such model calculations. The fraction of $\mathrm{D}$ in $O_{h^{-}}$and $T_{h}$-like sites changes from $(87 \%, 13 \%)$ at $25 \mathrm{~K}$ to $(81 \%, 19 \%)$ at 45
$\mathrm{K}$. When we plot the relative fraction of $T_{h}$ occupancy in an Arrhenius plot (Fig. 3), a straight line is obtained, corresponding to an excitation energy of 3.3 meV.

In Fig. 2 we also include the calculated D density for the two low-energy excited states in the potential of Fig. 2(b). The calculated excitation energies of the two states are 4 and $14 \mathrm{meV}$, respectively, i.e., of the same order of magnitude as the experimental value. Deuterium density contours [Figs. 2(d) and 2(e)] are clearly seen to be more $T_{h}$-like than those for the ground state [Fig. 2(c)], once again in qualitative agreement with the experimental findings. It can be seen from Fig. 2(e) that the 14-meV state has more D density spread out in the central region of the $\langle 100\rangle$ channeling axis than the $4-\mathrm{meV}$ state, and thus this state will give a more pronounced flux peak for the $\langle 100\rangle$ axial scan. The experimental results in Fig. 1 are thus most easily associated with the $14-\mathrm{meV}$ excited state, even though the calculated excitation energy of this state is somewhat larger than that obtained experimentally. In this connection, the approximate nature of the calculated $D$ potential should be kept in mind. It should also be pointed out that the coupling of the $\mathrm{D}$ motion to the lattice has not been included here. ${ }^{10}$

A very useful picture of the excitations discussed above can be obtained by consideration of the following analytically solvable model of the $\mathrm{D}$ motion in the vacancy. Assume that the D potential in Fig. 2(b) can be approximated by a spherically symmetrical potential $V(r)$. The wave function can then be written as $\psi_{n l m}(r)=\left[u_{n l}(r) / r\right] Y_{l m}(\theta, \phi)$, where $Y_{l m}$ are spherical harmonics, and the radial wave function satisfies the Schrödinger equation,

$$
\begin{aligned}
{\left[-\frac{\hbar^{2}}{2 M} \frac{d^{2}}{d r^{2}}\right.} & \left.+\frac{l(l+1) \hbar^{2}}{2 M r^{2}}+V(r)\right] u_{n l}(r) \\
& =\left[H_{0}+\frac{l(l+1) \hbar^{2}}{2 M r^{2}}\right] u_{n l}(r)=E_{n l} u_{n l}(r) .
\end{aligned}
$$

We shall make the further assumption that the excitation energies of the Hamiltonian $H_{0}$ are large compared to $l(l+1) \hbar^{2} / 2 M r^{2}$. This is well obeyed for small $l$ for the potential in Fig. 2, where $H_{0}$ approximately describes a harmonic oscillator situated a distance $R$ from the vacancy, with excitation energies $h \omega_{0} \sim 50 \mathrm{meV}$, whereas $\hbar^{2} / 2 M R^{2}=1.85 \mathrm{meV}$. In this case, we can treat the centrifugal potential using firstorder perturbation theory, and obtain the excitation spectrum which corresponds to the radial ground state, $E_{0 l}=\left[\hbar^{2} /\left(2 M R^{2}\right)\right] l(l+1)$, where $R$ is given by an expectation value of $1 / r^{2}$. In the case of $H_{0}$ describing a harmonic oscillator, $R$ is just the position of the minimum of the potential relative to the center of the vacancy. The only parameter determining the $\mathrm{D}$ exci- 

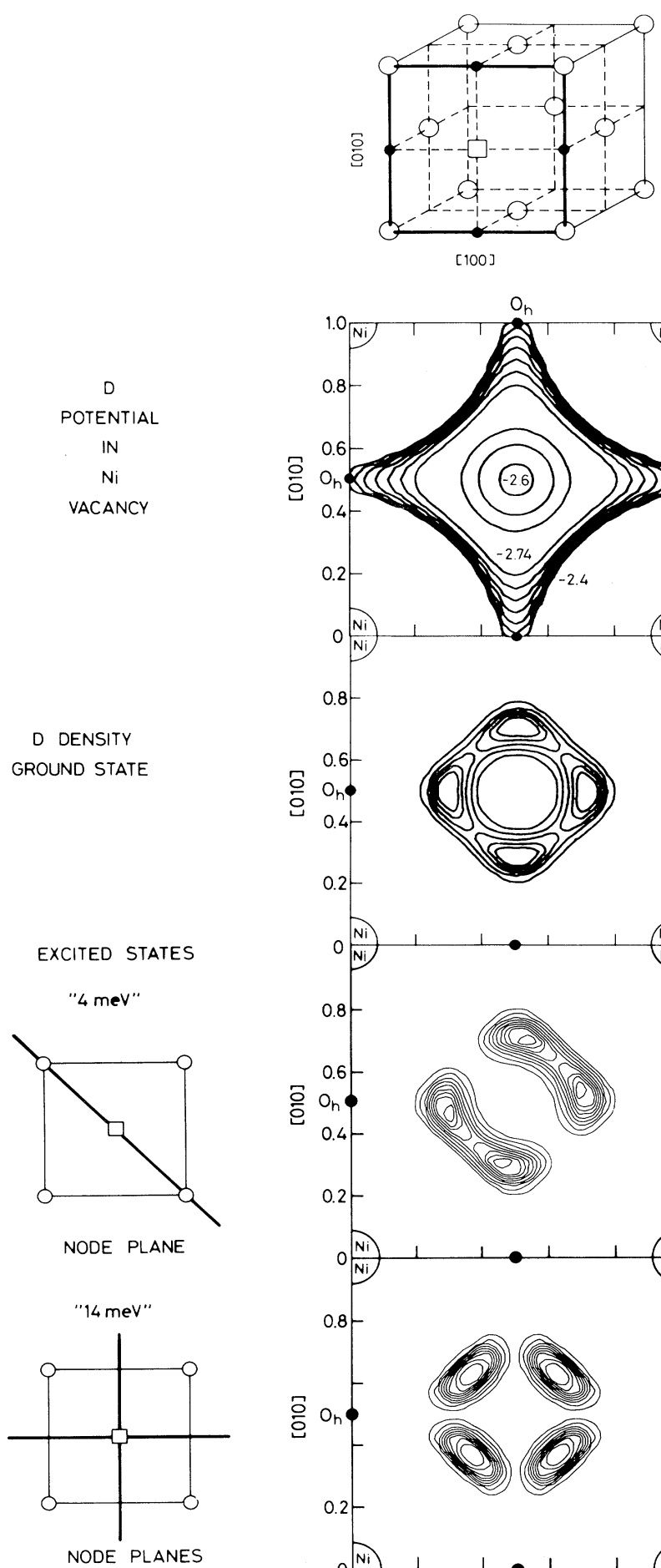
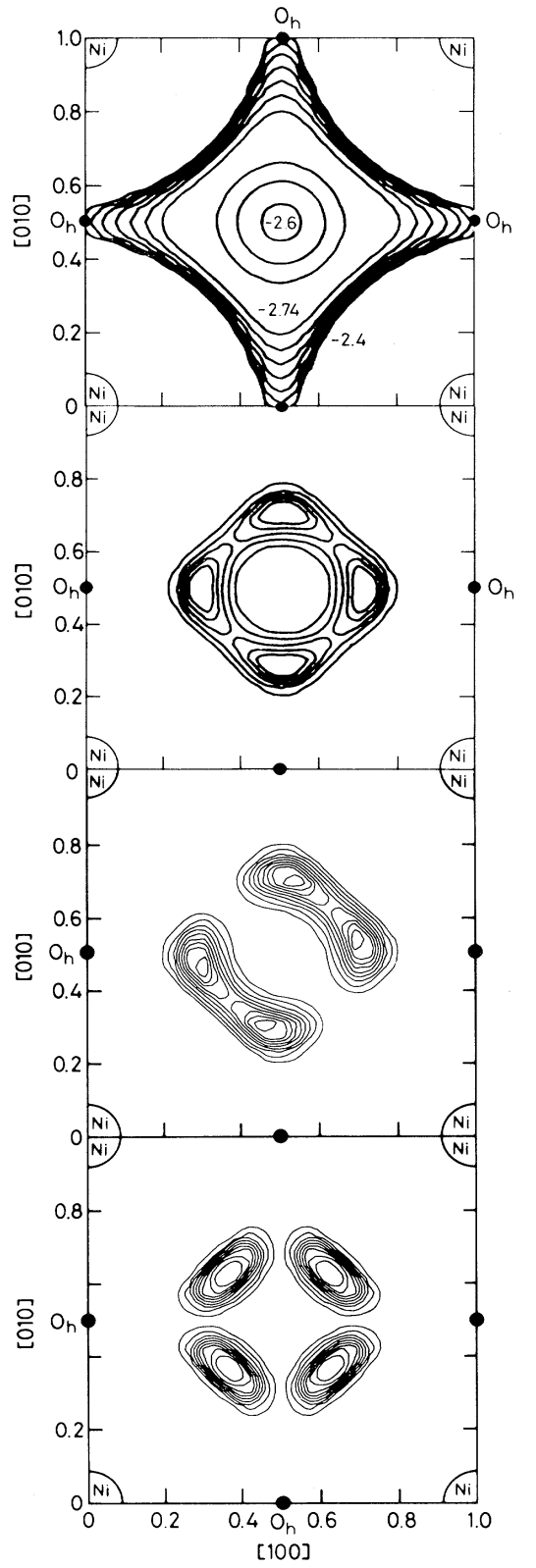

a)

Ni

$\square$ VACANCY

- $\mathrm{O}_{\mathrm{h}}$

- $T_{h}$

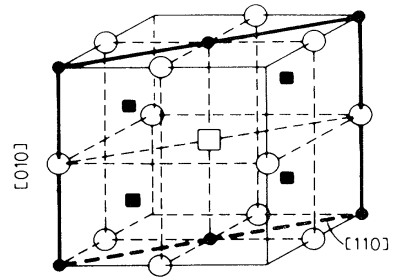

b)

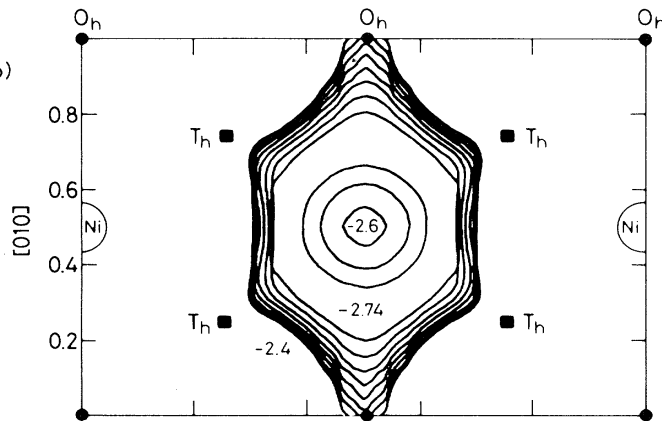

c)

d)

e)

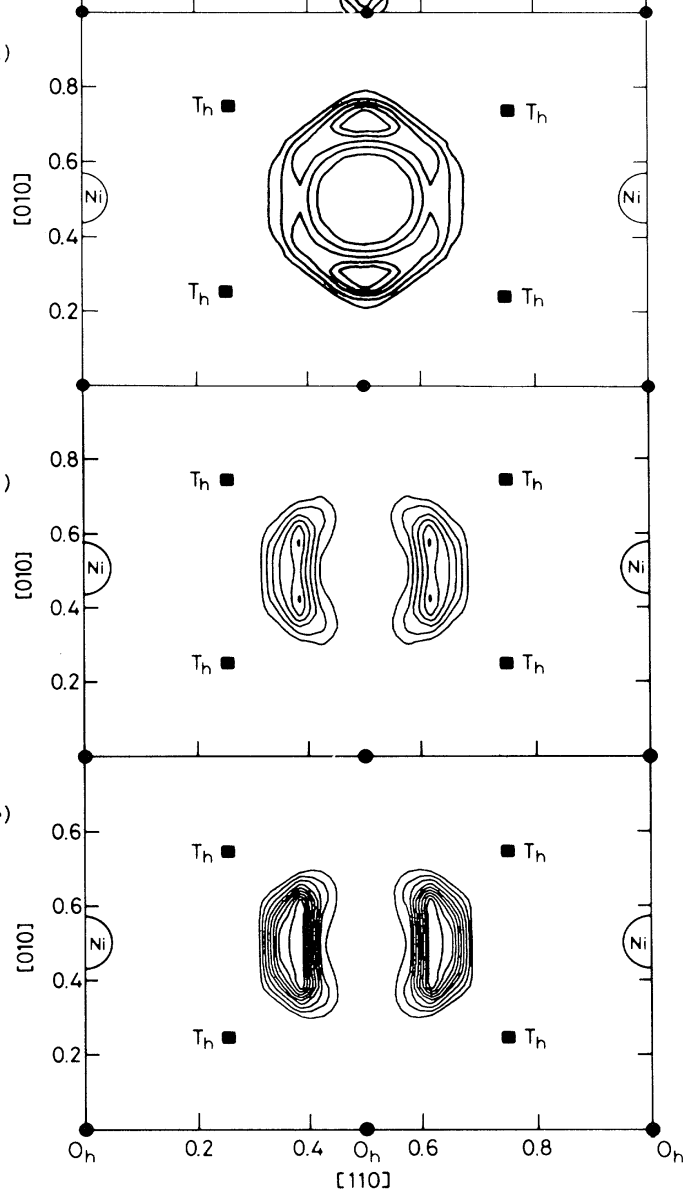

FIG. 2. Contour piots of the D potential (binding energy measured relative to a $D$ atom at $\infty$ ) and D density (wave function squared) for $\mathrm{D}$ in a $\mathrm{Ni}$ vacancy for two different cuts (accentuated by thick solid line) through the center of the vacancy. The contour spacing is $0.05 \mathrm{eV}$ in the potential plots and one sixth of the maximum in the density plots. From (a) it can be concluded that the projection of the $\langle 100\rangle$ axis on the (100) plane is only one fourth of the accentuated (100) frame. 


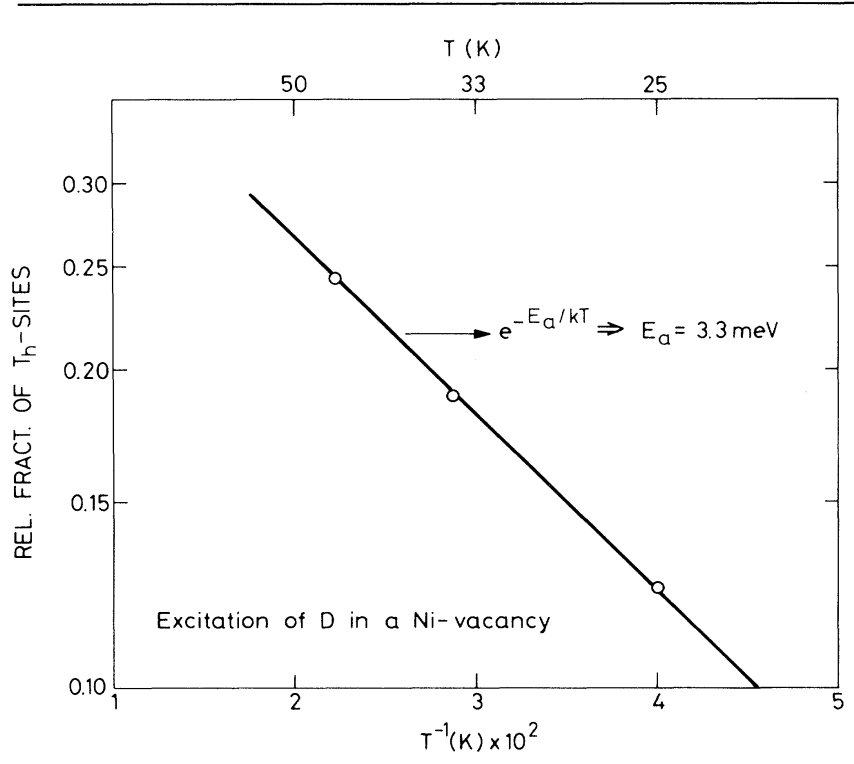

FIG. 3. The relative fraction of $T_{h}$ occupancy as a function of $1 / T(k)$.

tation spectrum is thus $R$, which can be read from Fig. 2. With $R=0.75 \AA$, the $l=1$ and $l=2$ excitation energies are 3.7 and $11.0 \mathrm{meV}$, respectively, very close to the results of the numerical solution in the full potential. The excited states are just the $p$ and $d$ states (standing waves) of a particle (D) moving on a sphere. The $p$ state has one mirror plane and the $d$ state two, as indicated in Fig. 2. ${ }^{11}$ Furthermore, if for $R$ we use the experimentally determined value $R=1.4 \AA$, we get excitation energies of 1.1 and $3.2 \mathrm{meV}$ for the $l=1$ and $l=2$ states, and thus the energy of the $l=2$ state is in very good agreement with the experimental excitation energy.

We would expect the effects described above for $\mathrm{Ni}$ also to be present in other hydrogen-metal systems. In the simple model, the only parameter describing the system is $R$, which scales approximately with the lattice constant, and which furthermore can be determined experimentally. For $\mathrm{Pd}$ and $\mathrm{Pt},{ }^{12}$ for example, the larger lattice constant causes an increase in $R$ and thereby a reduction of the excitation energies. This is in agreement with the experimental observations of a larger D fraction $(\sim 60 \%)$ in the delocalized $T_{h}$ site $(l=2)$ already at $25 \mathrm{~K}$ for these systems.

\footnotetext{
${ }^{1}$ In the present Letter, we deal only with the hydrogen isotope deuterium.

${ }^{2}$ F. Besenbacher, S. M. Myers, and J. K. Norskov, Nucl. Instrum. Methods Phys. Res., Sect. B 7/8, 55 (1985).

${ }^{3}$ S. M. Myers, W. R. Wampler, F. Besenbacher, S. L. Robinson, and N. R. Moody, Mater. Sci. Eng. 69, 397 (1985).

${ }^{4} J$. K. Norskov, F. Besenbacher, J. Bottinger, B. Bech Nielsen, and A. A. Pisarev, Phys. Rev. Lett. 49, 1420 (1982).
}

${ }^{5}$ M. S. Daw and M. I. Baskes, Phys. Rev. B 29, 6443 (1984).

${ }^{6}$ F. Besenbacher, H. Bogh, A. A. Pisarev, M. J. Puska, S. Holloway, and J. K. Norskov, Nucl. Instrum. Methods Phys. Res., Sect. B 4, 374 (1984).

${ }^{7}$ Materials Analysis by Ion Channeling, edited by L. C. Feldman, J. W. Mayer, and S. T. Picraux (Academic, New York, 1982).

8J. K. Nørskov, Phys. Rev. B 26, 2875 (1982), and Phys. Rev. Lett. 48, 1620 (1982); P. Nordlander, S. Holloway, and J. K. Norskov, Surf. Sci. 136, 59 (1984).

${ }^{9}$ M. J. Puska and R. M. Nieminen, Phys. Rev. B 29, 5382 (1984).

${ }^{10}$ Such effects can only be treated quantitatively in a dynamical treatment. Qualitatively, since the D excitation energy is lower than most phonon frequencies, the lattice will tend to follow the D motion. Such a situation can be described in terms of an increase in the effective mass of the impurity due to the drag of the metal atom (K. A. Muttalib and J. Sethna, to be published). The "static-lattice" excitation energy is therefore an upper bound on the real excitation energy.

${ }^{11}$ In the full numerical calculation, the $l=2$ states split up into $E_{g}$ and $T_{2 g}$ states. That which is shown in Fig. 2 is a $T_{2 g}$ state which is the one with most occupancy of the $T_{h}$ sites.

12J. P. Bugeat and E. Ligeon, Phys. Lett. 71A, 93 (1979); F. Besenbacher, B. Bech Nielsen, J. K. Nørskov, and M. J. Puska, to be published. 